\title{
Closing the gap in the capacity of random wireless networks ${ }^{1}$
}

\author{
Massimo Franceschetti ${ }^{2}$, Olivier Dousse ${ }^{3}$, David $_{\text {Tse }^{2}}$, Patrick Tiran ${ }^{3}$
}

\begin{abstract}
We consider the problem of how throughput in a wireless network with randomly located nodes scales as the number of users grows. Following the physical model of Gupta and Kumar, we show that randomly scattered nodes can achieve the optimal $1 / \sqrt{n}$ per-node transmission rate of arbitrarily located nodes. This contrasts with previous achievable results suggesting that a $1 / \sqrt{n \log n}$ reduced rate is the price to pay for the additional randomness introduced into the system. Our results rely on percolation theory arguments.
\end{abstract}

\section{INTRODUCTION}

A completely wireless network consists of $n$ nodes that communicate over a common wireless channel. A natural question that arises in such systems is how the throughput scales with the number $n$. This question was first addressed in [1]. These authors show that under the assumption that packets can be relayed from node to node and interference is simply regarded as noise at each hop, and if each node transmits to a destination non-vanishingly far away, the per-node throughput goes to zero at least as $1 / \sqrt{n}$ bit per second.

For random node locations and traffic patterns, they show that a per-node throughput of rate $1 / \sqrt{n \log n}$ can be achieved. Our contribution is to show that a per-node throughput rate of $1 / \sqrt{n}$ can also be achieved by networks of randomly located nodes, thus closing the gap between upper and lower bounds on the throughput capacity of such networks. Our proposed protocol also achieves the optimal average delay required for a packet to reach its destination.

\section{MAIN RESULT}

We consider a random network where nodes are distributed in a square of unit area according to a Poisson point process of intensity $n$. We let the distance between two nodes $i$ and $j$ be denoted by $d_{i j}$. We pick uniformly at random a matching of source-destination pairs, so that each node is the destination of exactly one source. Each node $i$ can transmit with any power $P_{i}$ less than the maximum power $P_{0}$, and node $j$ receives the transmitted signal with power $P_{i} L(i, j)$, where $L(\cdot)$ indicates the path loss between $i$ and $j$. We assume $L(i, j)=d_{i j}^{-\alpha} e^{-\gamma d_{i j}}$, for $\alpha>0, \gamma>0$, or $\alpha>2, \gamma=0$. We finally assume that two nodes can establish a wireless link of unit capacity if the signal to interference plus noise ratio at the receiver exceeds some threshold $t$. That is

$$
S I N R=\frac{P_{i} L(i, j)}{N_{0}+\sum_{k \neq i} P_{k} L(k, j)}>t
$$

\footnotetext{
${ }^{1} \mathrm{~A}$ complete version of this paper can be found at www. paradise. caltech. edu/ massimo/homepage/academic.html

${ }^{2}$ Dept. of Electrical Engineering and Computer Sciences, Univ. of California at Berkeley, Berkeley, CA 94720-1770. e-mail: \{massimof, dtse\}@EECS. berkeley.edu

${ }^{3}$ School of Computer and Communication Sciences EPFL CH-1015, Lausanne, Switzerland. e-mail: \{Patrick.Thiran, olivier.dousse\}@epfl.ch
}

where $N_{0}$ is the ambient noise power at the receiver. We say that in our stochastic model an event occurs with high probability (w.h.p.) if its probability tends to one as $n \rightarrow \infty$. The per-node throughput capacity of the network is defined as the number of bits per second that every node can transmit w.h.p. to its destination.

Our result is the following.

Theorem 1 The per-node throughput capacity of the network defined above is

$$
C(n)=\Theta(1 / \sqrt{n}) \text { bit } / \text { sec }
$$

\section{Percolation theory}

To prove our result we establish a connection between percolation theory [2] and wireless networks that can be potentially exploited to solve other information and network theoretic questions.

Let us consider the following example. Water is poured on one side of a large (ideally infinite) porous stone. What is the probability that the water finds a path to the opposite side? By modeling the stone as a square grid in which each edge can be open and hence traversed by water with probability $p$, and closed otherwise, independently of all other edges, one can show that for $p>1 / 2$ water percolates trough the stone with probability one. One can then ask at what rate the water percolates and how it depends on $p$. In other words, how rich in disjoint paths is the connected component of open edges? We relate this latter question to the information flow in our random wireless network. The open grid edges correspond to the presence of nodes in certain locations of the plane, and the open percolating paths represent the wireless backbone that is used to multi-hop packets across the network. We want to operate at $p>1 / 2$, above the percolation threshold, so that we can guarantee the existence of many paths that traverse the network, but also have $p<1$, so to avoid overcrowding and excessive interference. We show that controlling the parameter $p$ corresponds in a wireless network to scaling the transmission power at a given rate as $n \rightarrow \infty$. The scaling adopted in [1] corresponds to $p=1$, and to have a fully connected backbone containing paths that cross the network area. However, this wireless backbone covers all the nodes, generating excessive interference. By scaling the power at a higher rate, this backbone does not form at all. Percolation theory ensures that a different kind of backbone forms in the transition region between this two extreme scalings. This does not cover all the nodes, nevertheless it is sufficiently rich in crossing paths so that it can transport all the traffic in the network. By operating the network in this transition region between order and disorder, we are able to prove our tight bound.

\section{REFERENCES}

[1] P. Gupta, P. R. Kumar. "The capacity of wireless networks." IEEE Transactions on Information Theory, 46(2), 388-404, 2000.

[2] G. Grimmett. "Percolation." Second edition, Springer Verlag, 1999. 